\title{
A Preliminary Prospective Study of an Escalation in 'Maximum Daily Drinks', Fronto-Parietal Circuitry and Impulsivity-Related Domains in Young Adult Drinkers
}

\author{
Patrick D Worhunsky*,1, Alecia D Dager ${ }^{1,2,3}$, Shashwath A Meda², Sabin Khadka², Michael C Stevens ${ }^{1,2}$, \\ Carol S Austad ${ }^{3}$, Sarah A Raskin ${ }^{4}$, Howard Tennen ${ }^{5}$, Rebecca M Wood $^{3}$, Carolyn R Fallahi, \\ Marc N Potenza ${ }^{1,6,7,8}$ and Godfrey D Pearlson ${ }^{1,2,6,8}$
}

'Department of Psychiatry, Yale University School of Medicine, New Haven, CT, USA; ²Olin Neuropsychiatry Research Center, Hartford Hospitall Institute of Living, Hartford, CT, USA; ${ }^{3}$ Department of Psychology, Central Connecticut State University, New Britain, CT, USA; ${ }^{4}$ Department of Psychology, Trinity College, Hartford, CT, USA; ${ }^{5}$ Department of Community Medicine and Healthcare, University of Connecticut School of Medicine, Farmington, CT, USA; ${ }^{6}$ Department of Neurobiology, Yale University School of Medicine, New Haven, CT, USA; 7 Child Study Center, Yale University School of Medicine, New Haven, CT, USA

\begin{abstract}
Excessive alcohol use in young adults is associated with greater impulsivity and neurobiological alterations in executive control systems. The maximum number of drinks consumed during drinking occasions ('MaxDrinks') represents a phenotype linked to vulnerability of alcohol use disorders, and an increase, or 'escalation', in MaxDrinks may be indicative of greater risk for problematic drinking. Thirty-six young adult drinkers performed a Go/No-Go task during $\mathrm{MRI}$, completed impulsivity-related assessments, and provided monthly reports of alcohol use during a 12-month follow-up period. Participants were characterized by MaxDrinks at baseline and after follow-up, identifying 18 escalating drinkers and 18 constant drinkers. Independent component analysis was used to investigate functional brain networks associated with response inhibition, and relationships with principal component analysis derived impulsivity-related domains were examined. Greater baseline MaxDrinks was associated with an average reduction in the engagement of a right-lateralized fronto-parietal functional network, while an escalation in MaxDrinks was associated with a greater difference in fronto-parietal engagement between successful inhibitions and error trials. Escalating drinkers displayed greater impulsivity/compulsivity-related domain scores that were positively associated with frontoparietal network engagement and change in MaxDrinks during follow-up. In young adults, an escalating MaxDrinks trajectory was prospectively associated with altered fronto-parietal control mechanisms and greater impulsivity/compulsivity scores. Continued longitudinal studies of MaxDrinks trajectories, functional network activity, and impulsivity/compulsivity-related features may lend further insight into an intermediate phenotype vulnerable for alcohol use and addictive disorders.

Neuropsychopharmacology (2016) 4I, 1637-1647; doi:I0. I038/npp.2015.332; published online I8 November 2015
\end{abstract}

\section{INTRODUCTION}

Consumption of alcohol that exceeds recommended daily guidelines (eg, four drinks for women and five drinks for men; (NIAAA, 2004)), or 'binge drinking', in adolescents and young adults is linked to the development of alcohol use disorders (AUDs) that can persist into adulthood (Hill et al, 2000; McCarty et al, 2004), and chronic abuse of alcohol through the lifespan is associated with negative consequences that represent a substantial public health concern (Rehm et al, 2009). Binge drinking is common among young adults; however, most do not develop, or else remit from, an AUD

*Correspondence: Dr PD Worhunsky, Department of Psychiatry, Yale School of Medicine, I Church Street, Room 727, New Haven, CT 06519, USA, Tel: +I 203737 6473, Fax: + I 203737 359l, E-mail: patrick.worhunsky@yale.edu

${ }^{8}$ These authors contributed equally to this work.

Received 23 April 2015; revised 14 September 2015; accepted 14 September 2015; accepted article preview online 30 October 2015 by adulthood (Clark, 2004), suggesting that some young adult binge drinkers may exhibit specific vulnerabilities for developing a persistent AUD. The maximum number of drinks consumed on a single occasion ('MaxDrinks') is linked to the development of AUDs and may represent a reliable phenotype for identifying individuals at risk for AUDs (Grant et al, 2009; Saccone et al, 2000). An increase in the daily quantity consumed over time, or an 'escalation' in MaxDrinks, during early adulthood may further be indicative of a greater risk for developing AUDs (Auerbach and Collins, 2006; Schuckit et al, 2014). Investigating neurobiological and impulsivity-related features associated with an escalation in MaxDrinks in early adulthood may help identify vulnerable populations and inform prevention and intervention strategies.

Binge drinking in early adulthood is associated with alterations in executive control systems, including structural impairments in frontal brain regions (Jacobus and Tapert, 2013) and functional alterations (frequently observed as 
hyperactivity in prefrontal neural responses) related to working memory, decision making, and response inhibition (Petit et al, 2013). Less is known regarding the neurobiological features associated with an escalation in MaxDrinks in young adult drinkers. Adolescents who experience alcoholrelated blackouts (a possible indicator of high MaxDrinks drinking episodes) in early adulthood exhibit increased inhibition-related frontal activity relative to heavy drinking peers who do not experience blackouts (Wetherill et al, 2013). Similarly, young adults who escalate to heavy drinking relative to peers who maintain drinking levels, or 'constantdrinking' peers, display increased activity in frontal regions in response to alcohol-related cues (Dager et al, 2014). Direct examination of executive control mechanisms associated with increases in MaxDrinks may identify distinct neural correlates of AUD vulnerability.

Impairments in executive control mechanisms are associated with elevations in impulsivity and related domains (Bickel et al, 2012), which in turn are linked to the development and persistence of AUDs (Lejuez et al, 2010). Impulsivity is a multidimensional construct broadly encompassing behavioral dyscontrol associated with deficient inhibitory and/or aberrant motivational processes (Evenden, 1999). With respect to addictive disorders, impulsivity is increasingly discussed alongside compulsivity, another multidimensional construct of behavioral dyscontrol, but associated with repetitive, habitual, and often stereotyped behaviors (Fineberg et al, 2014). Dimensional analyses have identified an impulsivity-related domain integrating motivational aspect of impulsivity with compulsivity features-termed the 'impulsivity/compulsivity' domain - that is elevated in individuals with, or at risk for developing, addictive disorders (Castelluccio et al, 2014; Ginley et al, 2014; Hyatt et al, 2012; Meda et al, 2009; Patel et al, 2013). Impulsivity/compulsivity features are elevated in young adults with a family history of alcoholism and are linked with abnormal neural processing in reward circuitry (Andrews et al, 2011; Yarosh et al, 2014). Greater impulsivity/compulsivity is associated with increased alcohol-related consequences (ie, consequences possibly linked to high MaxDrinks drinking episodes) including blackouts, passouts, and feeling sick (Dager et al, 2014).

The current study investigated associations between MaxDrinks, executive control mechanisms involved in response inhibition, and impulsivity-related features in young adult drinkers. College students were assessed according to MaxDrinks at baseline and reclassified following 12 months of self-reported alcohol use as constant or escalating drinkers. We employed independent component analysis (ICA; (Calhoun et al, 2001)) to examine functional brain networks during baseline performance of a Go/No-Go response inhibition task, and used principal component analysis derived (PCA-derived) composite impulsivityrelated domain (CIRD) scores from multiple self-report questionnaires and behavioral tasks. We hypothesized that individuals who escalated in MaxDrinks relative to constant drinkers would exhibit a greater baseline engagement of networks encompassing fronto-parietal regions during a successful response inhibition. We also expected functional network engagement and prospective change in MaxDrinks would be linked to impulsivity-related domains, and in particular an impulsivity/compulsivity-related domain.

\section{MATERIALS AND METHODS}

\section{Participants}

Participants were 36 young adult drinkers recruited as part of the NIAAA-funded Brain and Alcohol Research in College Students (BARCS) research program (Ahmadi et al, 2013; Dager et al, 2014; Khadka et al, 2014). Individuals enrolled in the BARCS program provided a drinking history and completed a battery of psychometric tests including assessments of impulsivity-related domains. Participants submitted monthly reports of alcohol consumption, substance use, and other information through a secure website. Individuals were excluded from participating in fMRI procedures for a history of psychotic or bipolar disorder (assessed using the Mini International Neuropsychiatric Interview (MINI; (Sheehan et al, 1998)), significant brain injury, positive urine screen for illicit drugs or pregnancy, or any condition that would interfere with the ability to safely complete MRI procedures (eg, metallic implants and claustrophobia).

A subset of individuals in the BARCS program completed the Go/No-Go task during fMRI at baseline $(N=123)$. To address hypotheses related to prospective increases in MaxDrinks in young adult drinkers, individuals were excluded if they reported no lifetime drinking $(N=17)$ or did not complete at least one monthly report of alcohol use every quarter for the 12-month period following fMRI $(N=49)$. Additional participants were excluded for a high baseline MaxDrinks $(N=12)$ as individuals exhibiting a high MaxDrinks in early adulthood follow different MaxDrinks trajectories than their peers (Schuckit et al, 2014). For the purposes of this preliminary study, high MaxDrinks was defined as three or more drinks in excess of NIAAA guidelines for binge drinking episodes (NIAAA, 2004); ie, individuals with a baseline MaxDrinks of 7+ for females and $8+$ for males were excluded from analyses. Consistent with previous reports (Schuckit et al, 2014), the high MaxDrinks individuals averaged three times the MaxDrinks relative to non-high MaxDrinks participants (MaxDrinks $(\mathrm{SD})=10.3$ (3.2) and 3.1 (2.1), respectively) and averaged a decrease in MaxDrinks (average change $=-2.6 \quad(4.2)$ drinks) during follow-up. Of those excluded for insufficient follow-up reports, baseline interviews indicated over half would have been excluded from analyses for no history of alcohol use $(N=7)$ or a high baseline MaxDrinks $(N=25)$. Additional participants were excluded from analyses for meeting criteria for a current substance use disorder $(N=2)$ or moving excessively throughout fMRI $(N=7)$. The sample of 36 individuals represents a portion of participants included in an initial cross-sectional study (Ahmadi et al, 2013). Study procedures were approved by institutional review boards at Yale University, Hartford Hospital, Trinity College, and Central Connecticut State University, and participants provided written informed consent.

\section{Baseline Assessments and MaxDrinks Trajectory}

Baseline alcohol use (as well as histories of substance use, mood and anxiety disorders) was assessed using an in-house interview incorporating items from the Semi-Structured Assessment for the Genetics of Alcoholism (SSAGA; (Bucholz et al, 1994)), Structured Clinical Interview for the DSM-IV (SCID; (First et al, 2001)), and MINI (Sheehan et al, 
1998). Follow-up drinking behavior was assessed using the online monthly reports of drinking behavior for the 12 months following scanning. MaxDrinks was assessed with the question: 'What is the largest number of drinks containing alcohol that you have consumed in a 24-hour period...in the past 6-months (baseline)? or...in the past month (follow-up)?', and participants were provided standard drink units to assist in responding (ie, 'one drink equals: one 12-oz can or bottle of beer, one 5-oz glass of wine, one $12-\mathrm{oz}$ wine cooler, or $1 \mathrm{oz}$ of liquor straight or in a mixed drink').

Participants were median-split by past 6-month MaxDrinks $(0-3, \quad N=19,4-7, \quad N=17)$ to examine group differences at baseline. Individual MaxDrink trajectories were determined by comparing baseline MaxDrinks to the maximum Maxdrinks reported over the 12-month follow-up period. For the purposes of this preliminary study, 'escalating drinkers' were identified as participants displaying any increase in MaxDrinks during follow-up $(N=18)$, while 'constant drinkers' did not exceed baseline MaxDrinks during follow-up $(N=18)$.

\section{Go/No-Go Task}

Participants completed the Go/No-Go task described previously (Ahmadi et al, 2013; Steele et al, 2013; Stevens et al, 2007) during fMRI. Participants were instructed to press a button in response to all Go stimuli (' $\mathrm{X}$ ', presented at a variable ratio of $17: 20$ ) and withhold responses to No-Go stimuli (' $K$ ', presented at a variable ratio of $3: 20$ ). Stimuli were presented for $250 \mathrm{~ms}$ with pseudorandom intertrial intervals of 1,2 , or $3 \mathrm{~s}$. No-Go stimuli were presented $\sim 8-15 \mathrm{~s}$ apart. The task was administered in two runs of 246 trials, each lasting $\sim 7.5 \mathrm{~min}$. Task instructions emphasized speed and accuracy equally, and participants completed 10 practice trials before scanning. Responses within $1 \mathrm{~s}$ of stimulus onset were considered hits and errors for Go and No-Go trials, respectively.

\section{Image Acquisition and Processing}

Neuroimaging procedures were conducted on a Siemens Allegra 3T system (Siemens AG, Erlangen, Germany). Functional images were acquired using an echo planar image gradient echo pulse sequence covering the whole brain (TR/TE: $1500 / 28 \mathrm{~ms}$, flip angle: $65^{\circ}, \mathrm{FOV}: 24 \times 24 \mathrm{~cm}$, matrix: $64 \times 64, \quad 3.4 \times 3.4 \mathrm{~mm}$ in-plane resolution, $5 \mathrm{~mm}$ effective slice thickness, 30 slices). A total of 294 volumes were collected for each functional run, including an initial rest period of $9 \mathrm{~s}$ to allow for signal stabilization that was removed before image processing. Functional images were spatially processed using SPM8 (Wellcome Functional Imaging Laboratory, London, UK). Images were corrected for differences in slice acquisition time, and each run was independently realigned, normalized into Montreal Neurological Institute space at an isometric voxel size of $3 \times 3 \times 3 \mathrm{~mm}$, and smoothed with a 6-mm FWHM Gaussian kernel. Seven runs (from four escalating and three constant drinkers) with motion in excess of $4.5 \mathrm{~mm}$ or $3^{\circ}$ were excluded from subsequent analysis, leaving a total of 65 Go/No-Go runs across the 36 participants. All significant observations survived subsequent analyses that excluded all data from participants with excessive motion in a single run (ie, $N=29$ ).

\section{Independent Component Analysis}

ICA was performed as previously (Stevens et al, 2007) using the Group ICA of fMRI Toolbox (GIFT v2.0e; http://icatb. sourceforge.net). The dimensionality of the data set was estimated using minimum description length (MDL) criteria (Li et al, 2007). A total of 45 (ie, MDL estimate mean [17] +maximum [28]) components were extracted as higherorder ICA procedures improve the spatio-temporal quality of components (Abou-Elseoud et al, 2010). Analysis involved the concatenation of data into a single group that was reduced through a two-stage PCA. Components were extracted from the group aggregate using neural network algorithms designed to maximize independence of network outputs (Bell and Sejnowski, 1995). This process was iterated 20 times using ICASSO (Himberg et al, 2004) to assess stability and consistency of component extraction. Individual component time courses were reconstructed and scaled to percent BOLD signal change to facilitate comparisons between participants (Calhoun et al, 2001).

The resulting independent components represent functional brain networks, or systems of brain regions displaying temporally coherent activity. Multiple regression analysis of network time courses with the modeled hemodynamic activity associated with task event onsets (ie, Go-hits, No-Go correct omissions, and No-Go commission errors) produced $\beta$-weights used to identify networks associated with task events. This process is similar to traditional GLM-based analysis of fMRI data; however, instead of comparing the modeled activity with the measured BOLD signal in each voxel, the model is compared with the time course for each network (ie, a component of the measured BOLD signal that is observed in the source regions comprising a functional network). The resulting $\beta$-weights thus indicate the strength of association of each network with each task event. These $\beta$-weights represent a measure of 'engagement' or 'recruitment' of each network during task performance (Calhoun et al, 2001). One-sample $t$-tests identified 11 out of the 45 networks relating to correct omissions $\left(P_{\mathrm{FDR}}<0.001\right)$. Subsequent paired $t$-tests identified 3 out of the 11 networks that were differentially engaged by correct omissions (ie, successful response inhibition) as compared with commission errors $\left(P_{\mathrm{FDR}}<0.05\right)$.

\section{Composite Impulsivity-Related Domains}

At baseline, participants completed a battery of impulsivityrelated assessments including the Barratt Impulsiveness Scale (BIS-11; (Patton et al, 1995)), Behavioral Inhibition/Activation System scale (BIS/BAS; (Carver and White, 1994)), Padua Inventory (Sanavio, 1988), Sensitivity to Punishment and Sensitivity to Reward Questionnaire (SPSRQ; (Torrubia et al, 2001)), Zuckerman Sensation-Seeking Scale (ZSSS; (Zuckerman and Neeb, 1979)) Balloon Analog Risk Task (BART; (Lejuez et al, 2002)), and experiential discounting task (EDT; (Reynolds and Schiffbauer, 2004)). Impulsivityrelated domain scores were derived from a PCA performed on a larger sample of BARCS participants $(N=440)$. Consistent with research indicating dimensional constructs 
of impulsivity associated with substance-related and addictive disorders (Castelluccio et al, 2014; Ginley et al, 2014; Hyatt et al, 2012; Meda et al, 2009; Patel et al, 2013)), six impulsivity-related domains were identified within the 18 subscale and total scores. A full report of the PCA analysis is currently under review (Khadka et al, under review); however, additional details of the PCA analysis are provided in Supplementary Material.

\section{Statistical Analysis}

Analyses of network engagement were performed using repeated measures (success $\times$ error) analysis of variance (ANOVAs) with baseline- and prospective-drinking group factors. Post-hoc repeated measures analyses of identified main effects of prospective-drinking on network engagement were performed with nuisance covariates to control for potential influences of baseline drinking measures (ie, weeks and days per week drinking). Statistical analyses of impulsivity-related domains were performed using multivariate (to examine global differences in impulsivity) and univariate (to examine specific differences related to the impulsivity/compulsivity domain) ANOVAs with baselineand prospective-drinking group factors. Exploratory correlational analyses were conducted to examine relationships between MaxDrinks and change in MaxDrinks as continuous measures, network engagement metrics and impulsivityrelated domain scores.

\section{RESULTS}

\section{Participants, Task Performance, and Drinking Behavior}

Participant characteristics, task performance measures, and drinking behaviors are listed in Table 1. Participants did not differ demographically or in frequencies of lifetime and family history of AUDs, marijuana and tobacco use or lifetime depressive disorders (no participants met criteria for other Axis-I conditions) relative to baseline- or prospectivedrinking category. Participants performed similarly on the Go/No-Go task and completed a comparable percentage of monthly surveys during follow-up. Go/No-Go performance did not differ from never-drinkers or baseline high MaxDrink drinkers (ie, participants excluded from ICA; $P$ 's $>0.2$ ) and was similar to healthy adolescents and adults in previous studies using the same task (Steele et al, 2013; Stevens et al, 2007). Baseline groups (0-3 MaxDrinks as compared with 4+ MaxDrinks) did not differ in follow-up MaxDrinks or change in MaxDrinks during follow-up ( $P$ 's $>0.1$; Table 1$)$; and baseline MaxDrinks was not correlated with follow-up change in MaxDrinks $(r=-0.07$, $P=0.67)$.

Half of all participants exhibited some escalation in MaxDrinks during the 12-month follow-up. There was no difference in escalating-drinking relative to baseline mediansplit MaxDrinks groups $\left(\chi^{2}=0.11, P=0.74\right)$, with 10 of the 19 young adult drinkers with $0-3$ MaxDrinks at baseline increasing in MaxDrinks during follow-up, and 8 out of the 17 participants with 4+ MaxDrinks at baseline increasing in MaxDrinks during follow-up. Escalating drinkers did not differ from constant drinkers on baseline measures of drinking behavior (ie, number of weeks in the past 6-months, days drinking per week, and MaxDrinks; $P$ 's $>0.2$, Table 1). Escalating drinkers drank more regularly/frequently during follow-up than constant drinkers (i.e, percent months and days per month; $P^{\prime}$ s $<0.01$, Table 1 ).

\section{Functional Brain Networks}

ICA identified three components representing distinct networks of functionally coherent brain activity that were associated with successful response inhibition during Go/No-Go performance. The regional integration and taskrelatedness of each component are displayed in Figure 1a-c and a detailed list of the clusters comprising each component is provided in Table 2 .

A right-lateralized fronto-parietal network (Figure 1a) was more positively engaged during successful response inhibition than during commission errors across all participants $\left(\mathrm{F}_{1,33}=15.48, \quad P<0.001\right)$. There was a main effect of prospective-drinking group on within-subject fronto-parietal engagement, with escalating compared with constant drinkers exhibiting a greater difference in engagement between successful inhibitions and commission errors $\left(\mathrm{F}_{1,33}=5.77\right.$, $P=0.022)$. Success- and error-related $\beta$-weights did not differ between prospective-drinking groups $\left(P^{\prime} s>0.07\right)$. Prospective-drinking group differences in within-subject network engagement survived post-hoc tests that included nuisance covariates for baseline drinking measures (i.e, baseline MaxDrinks, weeks drinking and days per week drinking; $P<0.026$ ). There was a main effect of baseline drinking group on within-subject fronto-parietal network engagement with the 4+ MaxDrinks group exhibiting a greater difference in engagement between successful inhibitions and commission errors $\left(\mathrm{F}_{1,33}=4.81 ; P=0.035\right)$. There was also a main effect of baseline group on between-subject network engagement (ie, the average engagement associated with both successes and errors) with 4+ MaxDrinks group displaying a reduced average fronto-parietal engagement relative to the 0-3 MaxDrinks group $\left(\mathrm{F}_{1,33}=8.59, P=0.006\right)$. Greater fronto-parietal engagement during successful inhibition relative to commission errors was associated with faster overall reaction times $(r=-0.43, P=0.009)$ and increased commission error rates $(r=0.42, P=0.012)$.

An anterior dominant default mode network (Figure $1 \mathrm{~b}$ ) was more negatively engaged (or more 'disengaged') during successful response inhibition than commission errors across all participants $\left(\mathrm{F}_{1,33}=7.85, P=0.008\right)$. There were no main effects of prospective or baseline drinking group on withinor between-subject engagement of the default mode network $\left(\mathrm{F}_{1,33}\right.$ 's $\left.<0.4, P^{\prime} \mathrm{s}>0.5\right)$. Greater default mode network disengagement during successful inhibition relative to commission errors was associated with faster overall reaction times $(r=0.46, P=0.005)$ and increased commission error rates $(r=-0.61, P<0.001)$.

A temporo-occipito-parietal network (Figure 1c) was more positively engaged during successful response inhibition than commission errors across all participants $\left(\mathrm{F}_{1,33}=8.37\right.$, $P=0.007)$. There were no main effects of prospective or baseline drinking group on within- or between-subject engagement of the temporo-occipito-parietal network $\left(\mathrm{F}_{1,33}\right.$ 's $<0.5, \quad P$ 's $\left.>0.5\right)$. Greater network engagement during successful response inhibition relative to commission errors was associated with slower overall reaction 
Table I Participant Characteristics, Task Performance, and Drinking Behavior

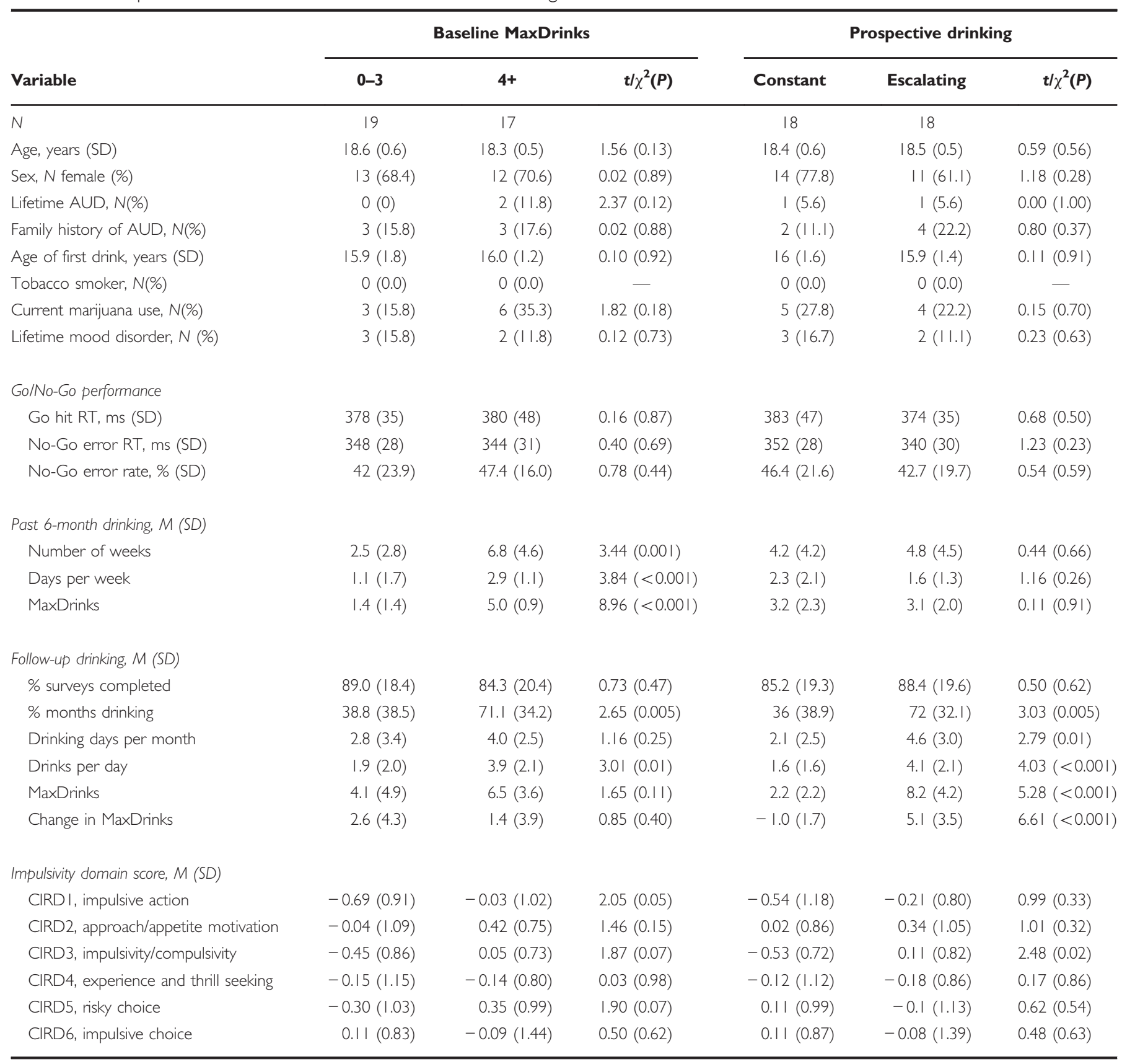

Abbreviations: AUD, alcohol use disorder; RT, reaction time.

times $(r=0.34, P=0.044)$ and reduced commission errors $(r=-0.41, P=0.012)$.

\section{Impulsivity-Related Domains}

Identified CIRD scores by baseline and prospective-drinking groups are listed in Table 1. PCA on 440 young adults in the larger BARCs study identified six domains: CIRD1, impulsive action (BIS-11 attentional, motor and nonplanning subscales, ZSSS disinhibition); CIRD2, approach/ appetite motivation (BIS/BAS drive, fun-seeking, and reward-responsivity subscales); CIRD3, impulsivity/compulsivity (SPSRQ reward and punishment sensitivity scores and
Padua compulsivity score); CIRD4, experience and thrill seeking (ZSSS thrill/adventure-seeking, experience-seeking, BIS/BAS inhibition); CIRD5, risky choice (BART total pumps, total explosions and adjusted total pumps); and CIRD6, impulsive choice (EDT area under the curve total score).

Multivariate analyses revealed a main effect of baseline drinking group $\left(\mathrm{F}_{6,28}=3.15, P=0.018\right)$, with the $4+$ MaxDrinks group relative to the $0-3$ MaxDrinks group exhibiting greater impulsivity across domain scores. A main effect of prospective-drinking group across the six impulsivity-related domains did not reach significance $\left(\mathrm{F}_{6,28}=2.02, P=0.096\right)$. Given specific hypothesis regarding the impulsivity/ 


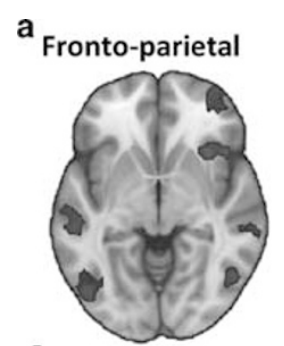

$-5$

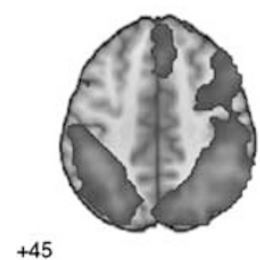

\section{$b_{\text {Default-mode }}$}

$-5$
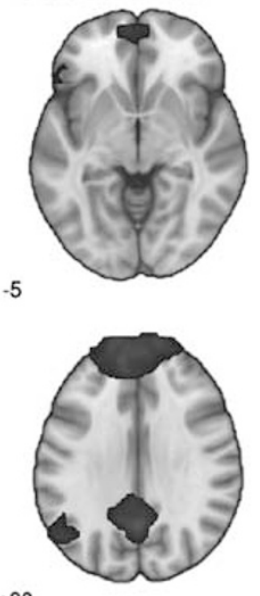

$+30$

${ }^{\text {C Temporo-occipito-parietal }}$

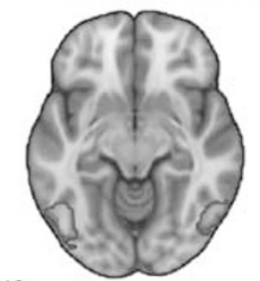

$-10$
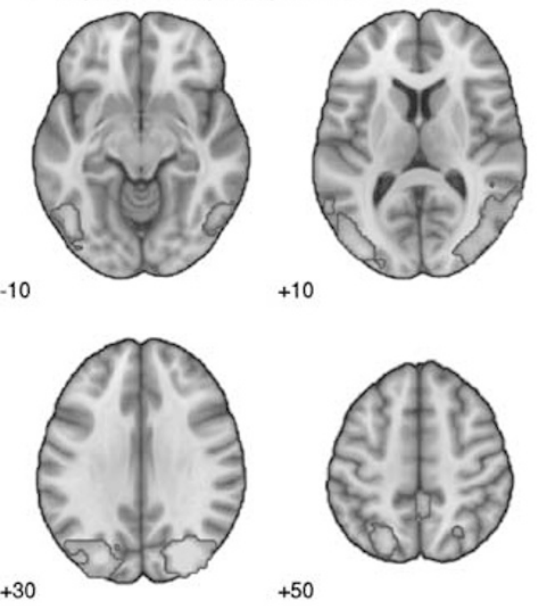

$+60$

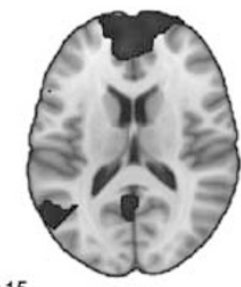

$+15$

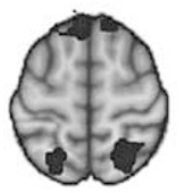

$+60$

$+10$

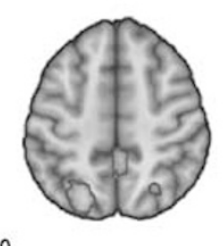

$+30$

$+50$

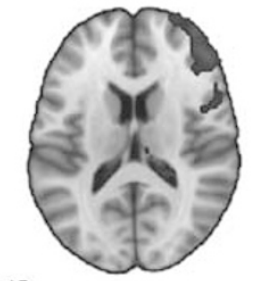

$+15$
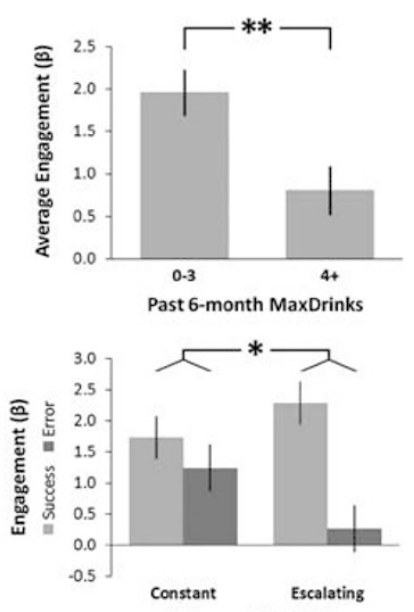

Follow-up MaxDrinks

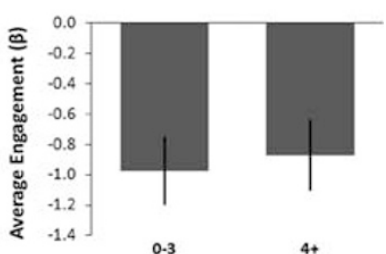

Past 6-month MaxDrinks

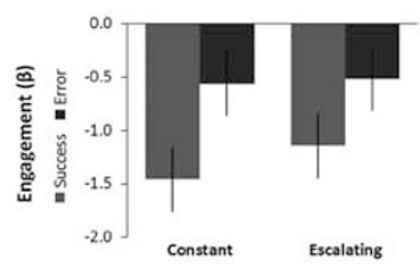

Follow-up MaxDrinks
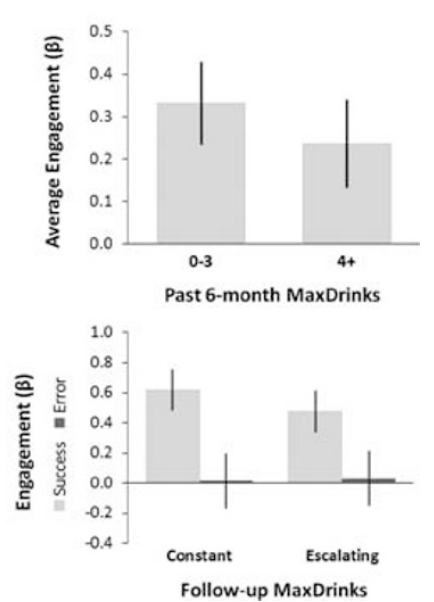

Figure I Regional integration of functional networks related to successful response inhibition displayed at cluster-corrected $P_{\mathrm{FWE}}<0.0 \mathrm{I}$ (voxelwise $P_{\text {uncorrected }}<0.00 \mathrm{I}$ ). Bar graphs display task-related engagement ( $\beta$-weight) associated with average (success+error) or differential (success, error) by baseline and prospective-drinking group (error bars indication SEM). A right-lateralized fronto-parietal network (a) was on average lower in the 4+ MaxDrinks group relative to 0-3 MaxDrinks groups at baseline $(* * P<0.01)$, while escalating drinkers as compared with constant drinkers exhibited a greater difference in network engagement between successful and error trials ( $P<0.05)$. Default mode (b) and temporo-occipito-parietal (c) networks did not differ between baseline or prospective-drinking groups. 
Table 2 Regional Integration of Functional Networks Associated with Response Inhibition

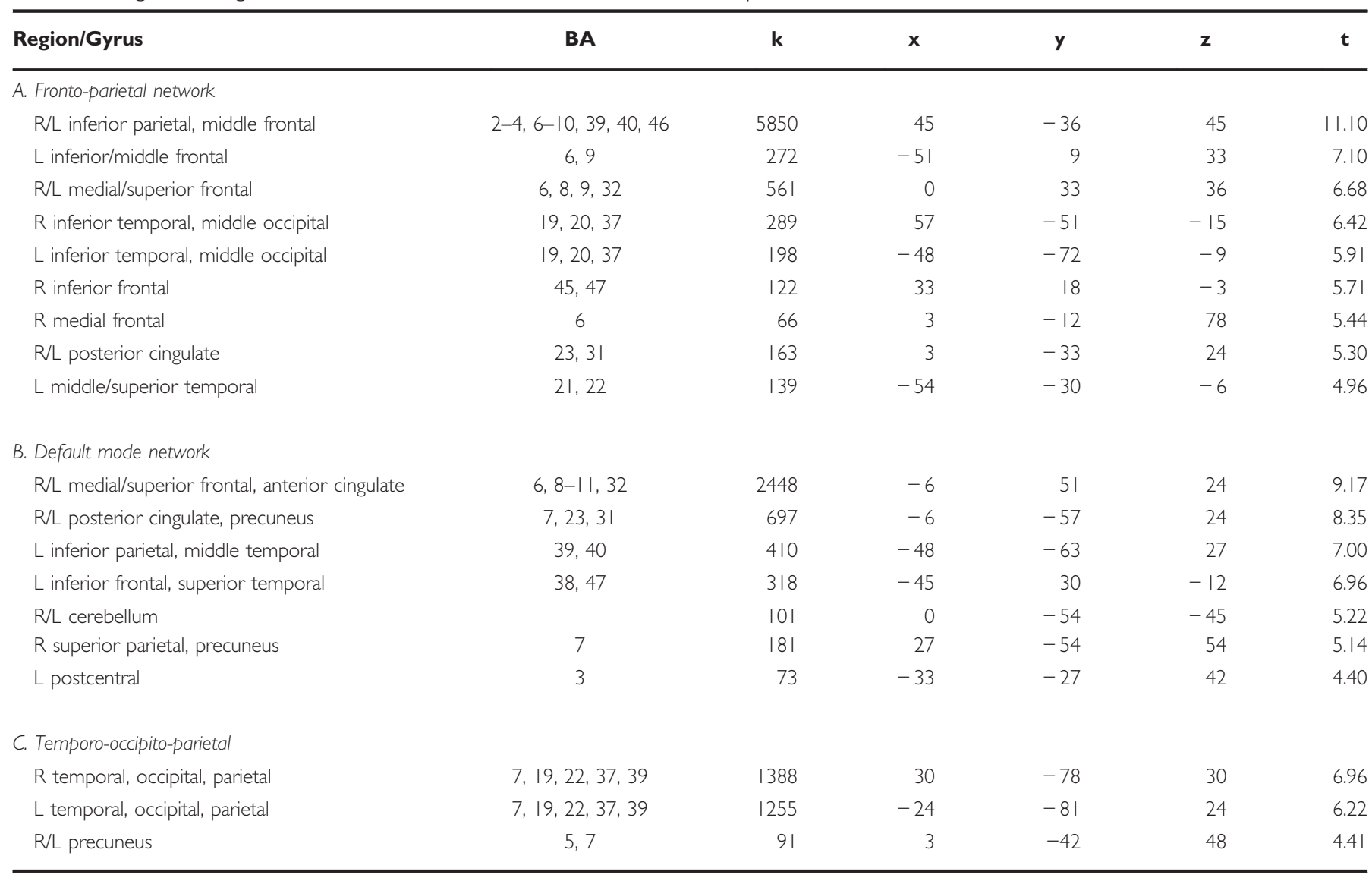

Abbreviations: BA, Brodmann areas; R/L, right/left hemisphere.

Details of functional network spatial source integration at cluster-corrected $P_{F W E}<0.0$ I (voxelwise $P<0.00 I$ ). Cluster information includes peak location $(x, y, z)$ in Montreal Neurological Institute space, $t$-score $(t)$, spatial extent ( $k$, in voxels), and associated BA.

compulsivity domain, additional analyses examining prospective group differences in CIRD3 revealed that escalating as compared with constant drinkers displayed greater impulsivity/compulsivity scores $\left(t_{1,32}=2.48, \quad P=0.018\right.$; Table 1), while a difference in impulsivity/compulsivity between baseline MaxDrinks groups did not reach significance $(P=0.07$; Table 1$)$.

\section{Exploratory Correlational Analyses}

As groups were defined using convenience criteria, correlational analyses were performed to explore relationships between baseline and follow-up MaxDrinks, fronto-parietal engagement, and impulsivity-related domain scores. Consistent with observed group differences, baseline MaxDrinks was negatively correlated with average (success +error) fronto-parietal engagement $(r=-0.42, P=0.011$; Figure 2a); however, there was no association between baseline MaxDrinks and differential (success - error) frontoparietal engagement $(r=0.2, P>0.3)$. Impulsivity/compulsivity domain scores did not correlate with baseline MaxDrinks $(P>0.1)$. Excluding one participant with a follow-up change in MaxDrinks 3 SD greater than the group average, change in MaxDrinks was correlated with differential fronto-parietal engagement $(r=0.41, \quad P=0.014$; Figure 2b), consistent with group analyses. Impulsivity/ compulsivity scores were positively associated with follow-up change in MaxDrinks ( $r=0.42, P=0.012$; Figure $2 \mathrm{c})$. Finally, impulsivity/compulsivity scores were positively associated with differential fronto-parietal engagement across all participants $(r=0.52, P=0.001$; Figure $2 \mathrm{~d})$.

\section{DISCUSSION}

The current study investigated functional brain networks associated with executive control during response inhibition and impulsivity-related domains in young adult drinkers who escalated in maximum number of drinks consumed on a single occasion, or 'MaxDrinks', relative to peers who remained at constant MaxDrinks drinking levels during a 12-month follow-up. Consistent with hypotheses, escalating drinkers relative to constant drinkers exhibited a greater difference in engagement of a fronto-parietal attentional/ control network during successful inhibition as compared with commission errors. This difference in fronto-parietal engagement was associated with the difference in MaxDrinks from baseline to follow-up and with impulsivity/compulsivity domain scores. The results from this initial study suggest an escalation in MaxDrinks may be associated with alterations in executive control mechanisms at a crucial period of AUD development. Associations between escalating MaxDrinks, functional network activity and impulsivity/ 

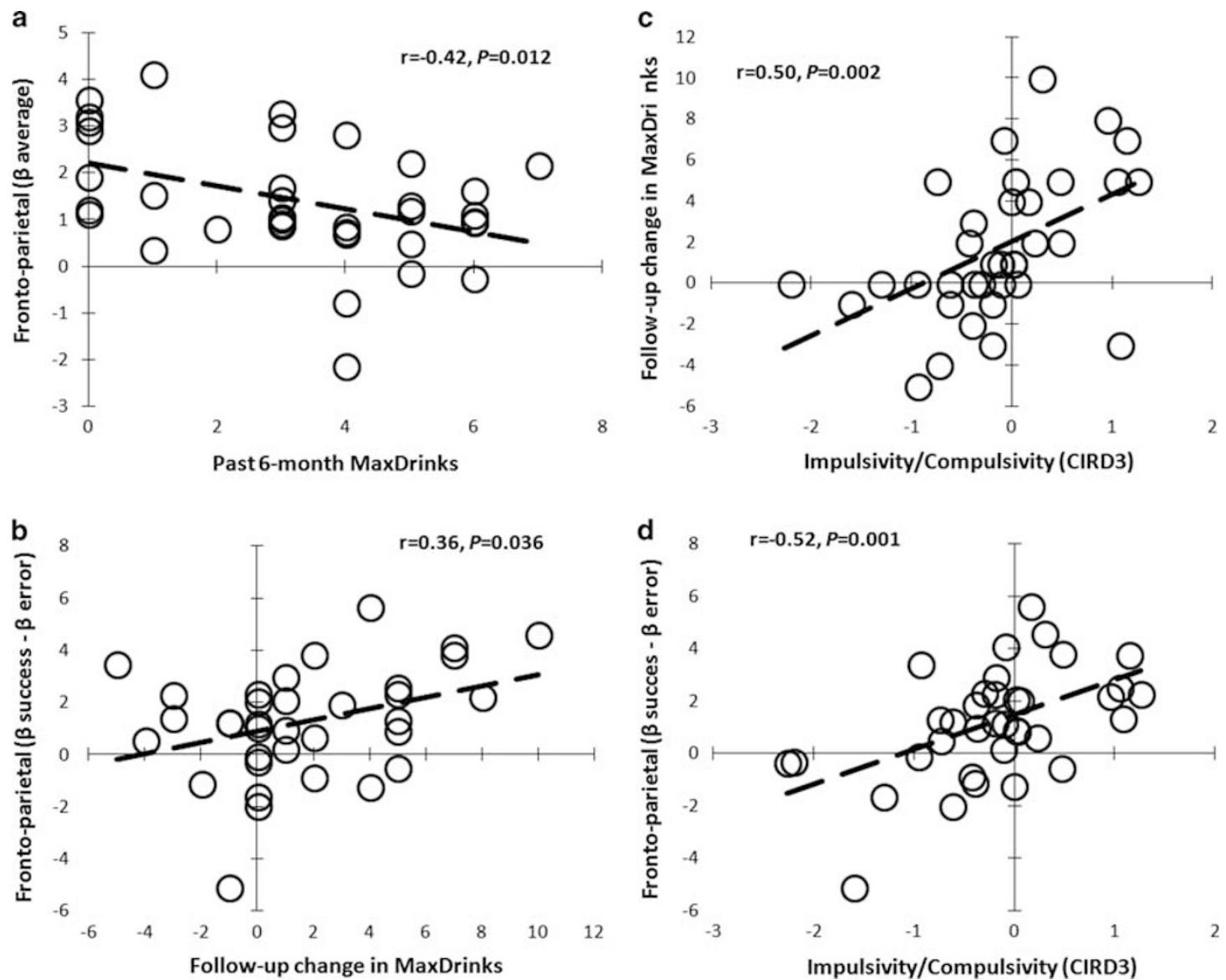

Figure 2 Exploratory correlational analyses between primary measures. (a) Average fronto-parietal engagement (success+error) was negatively correlated with baseline MaxDrinks $(r=-0.42, P=0.01 \mathrm{I})$. (b) Differential fronto-parietal engagement (success - error) was positively correlated with change in MaxDrinks during follow-up $(r=0.41, P=0.014)$. Impulsivity/compulsivity scores were positively correlated with (c) change in MaxDrinks during follow-up $(r=0.50, P=0.002)$, and $(d)$ with differential fronto-parietal engagement $(r=0.52, P=0.00 \mathrm{I})$.

compulsivity-related factors suggest an intermediate phenotype relating to vulnerability of developing persistent AUDs.

\section{Response Inhibition and Escalating MaxDrinks}

Inhibitory executive control in adolescent and young adult drinkers is of particular interest in understanding the acquisition and persistence of AUDs into adulthood (López-Caneda et al, 2013). Consistent with previous findings (Stevens et al, 2007), ICA identified three functional brain networks associated with successful response inhibition. The fronto-parietal, default mode, and temporooccipito-parietal networks identified were largely consistent with previously identified networks (Stevens et al, 2007), although differences in regional integration may be attributed to the higher dimensional ICA reduction method (Abou-Elseoud et al, 2010) used in the current study. All identified networks were significantly related to task performance measures.

Increased fronto-parietal engagement during successful response inhibition in young adult escalating drinkers is consistent with research in substance-naive adolescents who transitioned to heavy episodic drinking and experience blackouts in early adulthood (Wetherill et al, 2013). Engagement of the right-lateralized fronto-parietal network has been linked not only to response inhibition but also several other executive functioning domains including attention, memory, and decision making (Laird et al, 2011). As an executive control system, the fronto-parietal network may mediate activity between goal-directed, taskdependent processes and internal, default mode processes (Chen et al, 2013). This effective relationship of frontoparietal activity on default mode processes appears underdeveloped in adolescents during response inhibition (Stevens et al, 2007). Fronto-parietal hyper-engagement in escalating drinkers may thus reflect a global cognitive functioning impairment in that successful response inhibition requires an increased coordination of goal-directed and internal processes, while this mechanism may be more developed, or less impaired, in young adult constant drinkers.

Regarding MaxDrinks at baseline, a similar pattern of greater success - error differential engagement was observed in the 4+ MaxDrinks group relative to the 0-3 MaxDrinks group, although this difference was observed along with a blunted average (success+error) engagement of the frontoparietal network. This finding is in line with previous research associating binge drinking with increased responseinhibition-related activity in frontal and parietal regions (see (Petit et al, 2013)), and extends support for compensatory models of fronto-parietal hyperactivity in young binge 
drinkers (ie, increased demand-related activity is needed to overcome tonically deficient control mechanisms) (Scheurich, 2005). Information regarding the chronicity of drinking at MaxDrinks levels prior to baseline was not available in the current study; however, fronto-parietal impairments in young adult drinkers may reflect a combination of predisposing vulnerabilities and neural consequences of heavy drinking (Jacobus and Tapert, 2013).

There were no prospective or baseline drinking group differences in default mode or temporo-occipito-parietal network engagement during response inhibition. Default mode network alterations have been observed in adult alcoholics during resting state, although increased connectivity patterns may compensate for these deficiencies during task performance (Müller-Oehring et al, 2014). The temporo-occipito-parietal network may facilitate the integration of ventro-lateral and dorsal visual streams involved in stimuli shape detection (eg, ' $\mathrm{X}$ ' or ' $\mathrm{K}$ ' in the current Go/NoGo task) (Zachariou et al, 2014). The lack of MaxDrinksrelated differences in these networks in young drinkers suggests these mechanisms may be intact during response inhibition.

\section{Impulsivity-Related Domains and MaxDrinks Trajectories}

Young adult escalating drinkers relative to constant drinkers exhibited greater impulsivity-related domain scores linked to reward/punishment-sensitive impulsivity (SPSRQ sensitivity to punishment and sensitivity to reward scores) and compulsivity (Padua Inventory scores). The current finding of broadly elevated impulsivity-related features in baseline 4+ MaxDrink drinkers, but greater compulsivity-related features in escalating drinkers suggests the proposed transition from impulsive to compulsive tendencies in addictive disorders (Fineberg et al, 2014) may predate the acquisition of AUDs in some young adults. The reward/punishment-sensitive aspects of the impulsivity/compulsivity domain, as compared with similarly constructed but separately identified behavioral activation/inhibition domain, suggests an integration of motivational features related to specific rewards and consequences (Torrubia et al, 2001). The elevated impulsivity/ compulsivity in escalating drinkers is consistent with research linking this domain with increased frequencies of binge-drinking-related consequences in young adults (Dager et al, 2014), and research implicating this domain in other addictive disorders (Castelluccio et al, 2014; Ginley et al, 2014; Hyatt et al, 2012; Meda et al, 2009; Patel et al, 2013). Greater impulsivity/compulsivity in young adults may reflect a specific vulnerability for escalating-drinking behavior or addictive disorders more broadly, and additional longitudinal studies are needed to further explore the progression of impulsive and compulsive features in AUDs and addictions.

\section{MaxDrinks, Executive Control and Impulsivity/Compulsivity as an AUD Vulnerable Intermediate Phenotype}

MaxDrinks is linked to the development of AUDs and associated with genetic markers related to alcohol metabolism (Grant et al, 2009; Saccone et al, 2000), suggesting
MaxDrinks may be considered a phenotype related to AUD vulnerability. Similarly, as reviewed above, impulsivity/ compulsivity is linked to addictive disorders (eg (Meda et al, 2009)) and associated with genetic pathways involving brain development that may influence the maturation and organization of executive control systems (Khadka et al, 2014; Stevens et al, 2009). Future studies should examine the extent to which genetic mechanisms may influence the development of executive control (perhaps the frontoparietal system in particular) in relation to impulsivity/ compulsivity features that may predispose individuals to the development of addictive disorders.

In this initial study, while baseline MaxDrinks did not predict a follow-up change in MaxDrinks, fronto-parietal functioning was associated with both MaxDrinks at baseline and a follow-up change in MaxDrinks. Thus, alterations in executive control mechanisms (expressed as elevated impulsivity/compulsivity) may influence the risk associated with the relationships between MaxDrinks, escalatingdrinking, and AUDs. However, these possible vulnerability factors (ie, MaxDrink-related and executive-control-related) may not be entirely independent as adolescents with a family history of AUDs display reduced fronto-parietal functional connectivity and impaired prefrontal response inhibition processing relative to family history-negative peers (Hardee et al, 2014; Wetherill et al, 2012). Furthermore, as individuals displaying escalating-drinking patterns into young adulthood are more likely to develop non-alcohol substance use disorders (Hill et al, 2000), these impairments may not be specific to alcohol use, but perhaps addictive behaviors more broadly.

\section{Limitations and Future Directions}

The current study is limited by relatively small samples, and represents a preliminary investigation that requires replication in larger cohorts. Sample sizes limited the ability to investigate potential influences of multiple factors that may be associated with escalating-drinking, including gender, socioeconomic status, and pubertal timing, or investigate mutliple trajectories of MaxDrinks through young adulthood (Schuckit et al, 2014). Although baseline and prospectivedrinking categories were defined using convenience criteria relative to MaxDrinks, escalating drinkers relative to constant drinkers displayed increases in non-MaxDrinksrelated frequency of alcohol use (ie, months of drinking and days drinking per month). Thus group distinctions based on MaxDrinks alone may have captured a broader pattern of frequent heavy alcohol use, and perhaps tolerance-related influences on drinks consumed that may differ from escalating MaxDrinks as a risk behavior. Although functional network activity and impulsivity-related factors were not associated with follow-up drinking frequency metrics, data assessing tolerance were not available in the current study. MaxDrinks trajectories were investigated in a convenience sample of college student drinkers during a 12-month period from approximately age $18 / 19$ to age $19 / 20$ years. Future research is needed to examine the relationships of observed findings to the neural correlates and impulsivity-related features of non-drinkers, and MaxDrink trajectories in high MaxDrinks drinkers. 


\section{CONCLUSIONS}

Baseline MaxDrinks was associated with generally blunted fronto-parietal engagement and broadly elevated impulsivity-related domain scores. Individuals who increased in MaxDrinks during follow-up exhibited greater frontoparietal network engagement during response inhibition and increased impulsivity/compulsivity domain scores. Average fronto-parietal engagement (success+error) was negatively correlated with baseline MaxDrinks, while differential engagement (success - error) positively correlated with the change in MaxDrinks during follow-up. The current study is the first to demonstrate a relationship between altered frontoparietal control mechanisms and this impulsivity/compulsivity-related domain in young adult drinkers that is associated with a prospective escalation in MaxDrinks. Continued longitudinal studies of MaxDrinks trajectories, functional network activity and impulsivity/compulsivity-related domains may lend further insight into a vulnerability-related intermediate phenotype and inform intervention strategies.

\section{FUNDING AND DISCLOSURE}

GDP has been a consultant to Bristol Myers Squibb for projects unrelated to the current research. MNP has consulted for and advised Ironwood, Lundbeck, INSYS, Shire, and RiverMend Health; has received research support from Mohegan Sun Casino, the National Center for Responsible Gaming, and Pfizer; has participated in surveys, mailings, or telephone consultations related to drug addiction, impulse control disorders, or other health topics; has consulted for legal entities on issues related to impulse control and addictive disorders; provides clinical care in the Connecticut Department of Mental Health and Addiction Services Problem Gambling Services Program; has performed grant reviews for the National Institutes of Health and other agencies; has edited journals or journal sections; has given academic lectures in grand rounds, CME events and other clinical or scientific venues; and has generated books or book chapters for publishers of mental health texts. HT has received compensation from Wiley Publishers. The authors declare no conflicts of interest.

\section{ACKNOWLEDGMENTS}

We thank the administrative and technical support of Farah Aslanzadeh, Gregory Book, Scott Bullock and Jiansong $\mathrm{Xu}$, and the helpful comments provided by reviewers. This research was funded by NIAAA grant support (GDP: AA016599, AA019036, AA017539). Additional financial support was provided by NIAAA (AA015496) and NIDA (MNP: P20 DA027844) grants.

\section{REFERENCES}

Abou-Elseoud A, Starck T, Remes J, Nikkinen J, Tervonen O, Kiviniemi V (2010). The effect of model order selection in group PICA. Hum Brain Mapp 31: 1207-1216.

Ahmadi A, Pearlson GD, Meda SA, Dager A, Potenza MN, Rosen R et al (2013). Influence of alcohol use on neural response to go/nogo task in college drinkers. Neuropsychopharmacology 38: 2197-2208.
Andrews MM, Meda SA, Thomas AD, Potenza MN, Krystal JH, Worhunsky $\mathrm{P}$ et al (2011). Individuals family history positive for alcoholism show functional magnetic resonance imaging differences in reward sensitivity that are related to impulsivity factors. Biol Psychiatry 69: 675-683.

Auerbach KJ, Collins LM (2006). A multidimensional developmental model of alcohol use during emerging adulthood. J Stud Alcohol 67: 917-925.

Bell AJ, Sejnowski TJ (1995). An information-maximization approach to blind separation and blind deconvolution. Neural Comput 7: 1129-1159.

Bickel WK, Jarmolowicz DP, Mueller ET, Gatchalian KM, McClure SM (2012). Are executive function and impulsivity antipodes? A conceptual reconstruction with special reference to addiction. Psychopharmacology 221: 361-387.

Bucholz KK, Cadoret $\mathrm{R}$, Cloninger CR, Dinwiddie $\mathrm{SH}$, Hesselbrock VM, Nurnberger JI Jr et al (1994). A new, semistructured psychiatric interview for use in genetic linkage studies: a report on the reliability of the SSAGA. J Stud Alcohol 55: 149.

Calhoun V, Adali T, Pearlson G, Pekar J (2001). A method for making group inferences from functional MRI data using independent component analysis. Hum Brain Mapp 14: 140-151.

Carver CS, White TL (1994). Behavioral inhibition, behavioral activation, and affective responses to impending reward and punishment: the BIS/BAS scales. J Pers Soc Psychol 67: 319.

Castelluccio BC, Meda SA, Muska CE, Stevens MC, Pearlson GD (2014). Error processing in current and former cocaine users. Brain Imaging Behav 8: 87-96.

Chen AC, Oathes DJ, Chang C, Bradley T, Zhou Z-W, Williams LM et al (2013). Causal interactions between fronto-parietal central executive and default-mode networks in humans. Proc Natl Acad Sci USA 110: 19944-19949.

Clark DB (2004). The natural history of adolescent alcohol use disorders. Addiction 99: 5-22.

Dager AD, Anderson BM, Rosen R, Khadka S, Sawyer B, Jiantonio-Kelly RE et al (2014). Functional magnetic resonance imaging (fMRI) response to alcohol pictures predicts subsequent transition to heavy drinking in college students. Addiction 109: 585-595.

Evenden JL (1999). Varieties of impulsivity. Psychopharmacology 146: $348-361$.

Fineberg NA, Chamberlain SR, Goudriaan AE, Stein DJ, Vanderschuren LJ, Gillan CM et al (2014). New developments in human neurocognition: clinical, genetic, and brain imaging correlates of impulsivity and compulsivity. CNS Spectr 19: 69-89.

First MB, Spitzer RL, Gibbon M, Williams JB (2001). Structured Clinical Interview for DSM-IV-TR Axis I Disorders-Patient Edition (SCID-I/P. 2/2001 Revision). Biometrics Research Department, New York State Psychiatric Institute: New York.

Ginley MK, Whelan JP, Meyers AW, Relyea GE, Pearlson GD (2014). Exploring a multidimensional approach to impulsivity in predicting college student gambling. J Gambl Stud 30: 521-536.

Grant JD, Agrawal A, Bucholz KK, Madden PA, Pergadia ML, Nelson EC et al (2009). Alcohol consumption indices of genetic risk for alcohol dependence. Biol Psychiatry 66: 795-800.

Hardee JE, Weiland BJ, Nichols TE, Welsh RC, Soules ME, Steinberg DB et al (2014). Development of impulse control circuitry in children of alcoholics. Biol Psychiatry 76: 708-716.

Hill KG, White HR, Chung IJ, Hawkins JD, Catalano RF (2000). Early adult outcomes of adolescent binge drinking: person-and variable-centered analyses of binge drinking trajectories. Alcohol Clin Exp Res 24: 892-901.

Himberg J, Hyvärinen A, Esposito F (2004). Validating the independent components of neuroimaging time series via clustering and visualization. NeuroImage 22: 1214-1222.

Hyatt CJ, Assaf M, Muska CE, Rosen RI, Thomas AD, Johnson MR et al (2012). Reward-related dorsal striatal activity differences 
between former and current cocaine dependent individuals during an interactive competitive game. PloS One 7: e34917.

Jacobus J, Tapert SF (2013). Neurotoxic effects of alcohol in adolescence. Annu Rev Clin Psychol 9: 703-721.

Khadka S, Narayanan B, Meda S, Gelernter J, Han S, Sawyer B et al (2014). Genetic association of impulsivity in young adults: a multivariate study. Transl Psychiatry 4: e451.

Khadka S, Stevens MC, Aslanzadeh F, Broderick S, Narayanan B, Hawkins KA et al (under review). Composite impulsivity-related domains in young adults: a study on college students.

Laird AR, Fox PM, Eickhoff SB, Turner JA, Ray KL, McKay DR et al (2011). Behavioral interpretations of intrinsic connectivity networks. J Cogn Neurosci 23: 4022-4037.

Lejuez C, Magidson JF, Mitchell SH, Sinha R, Stevens MC, De Wit H (2010). Behavioral and biological indicators of impulsivity in the development of alcohol use, problems, and disorders. Alcohol Clin Exp Res 34: 1334-1345.

Lejuez C, Read JP, Kahler CW, Richards JB, Ramsey SE, Stuart GL et al (2002). Evaluation of a behavioral measure of risk taking: the Balloon Analogue Risk Task (BART). J Exp Psychol Appl 8: 75.

Li YO, Adali T, Calhoun VD (2007). Estimating the number of independent components for functional magnetic resonance imaging data. Hum Brain Mapp 28: 1251-1266.

López-Caneda E, Holguín SR, Cadaveira F, Corral M, Doallo S (2013). Impact of alcohol use on inhibitory control (and vice versa) during adolescence and young adulthood: a review. Alcohol Alcohol 49: 173-181.

McCarty CA, Ebel BE, Garrison MM, DiGiuseppe DL, Christakis DA, Rivara FP (2004). Continuity of binge and harmful drinking from late adolescence to early adulthood. Pediatrics 114: 714-719.

Meda SA, Stevens MC, Potenza MN, Pittman B, Gueorguieva R, Andrews MM et al (2009). Investigating the behavioral and selfreport constructs of impulsivity domains using principal component analysis. Behav Pharmacol 20: 390.

Müller-Oehring EM, Jung Y-C, Pfefferbaum A, Sullivan EV, Schulte T (2014). The resting brain of alcoholics. Cereb Cortex 25: $4155-4168$.

NIAAA (2004). NIAAA council approves definition of binge drinking. NIAAA Newsletter 3: 3.

Patel KT, Stevens MC, Meda SA, Muska C, Thomas AD, Potenza MN et al (2013). Robust changes in reward circuitry during reward loss in current and former cocaine users during performance of a monetary incentive delay task. Biol Psychiatry 74: 529-537.

Patton JH, Stanford MS, Barratt ES (1995). Factor structure of the Barratt impulsiveness scale. J Clin Psychol 51: 768-774.

Petit G, Maurage P, Kornreich C, Verbanck P, Campanella S (2013). Binge drinking in adolescents: a review of neurophysiological and neuroimaging research. Alcohol Alcohol 49: 198-206.

Rehm J, Mathers C, Popova S, Thavorncharoensap M, Teerawattananon Y, Patra J (2009). Global burden of disease and injury and economic cost attributable to alcohol use and alcohol-use disorders. Lancet 373: 2223-2233.

Reynolds B, Schiffbauer R (2004). Measuring state changes in human delay discounting: an experiential discounting task. Behav Processes 67: 343-356.

Saccone NL, Kwon JM, Corbett J, Goate A, Rochberg N, Edenberg HJ et al (2000). A genome screen of maximum number of drinks as an alcoholism phenotype. Am J Med Genet 96: 632-637.

Sanavio E (1988). Obsessions and compulsions: the Padua Inventory. Behav Res Ther 26: 169-177.

Scheurich A (2005). Neuropsychological functioning and alcohol dependence. Curr Opin Psychiatry 18: 319-323.

Schuckit MA, Smith TL, Danko GP, Bucholz KK, Agrawal A, Dick DM et al (2014). Predictors of subgroups based on maximum drinks per occasion over six years for 833 adolescents and young adults in COGA. J Stud Alcohol Drugs 75: 24.

Sheehan D, Lecrubier Y, Sheehan K, Amorim P, Janavs J, Weiller E et al (1998). The validity of Mini International Neuropsychiatric Interview (MINI). The development and validation of a structured diagnostic interview for DSM-IV and ICD-10. J Clin Psychiatry 59: 211-232.

Steele VR, Aharoni E, Munro GE, Calhoun VD, Nyalakanti P, Stevens MC et al (2013). A large scale $(\mathrm{N}=102)$ functional neuroimaging study of response inhibition in a Go/NoGo task. Behav Brain Res 256: 529-536.

Stevens MC, Kiehl KA, Pearlson GD, Calhoun VD (2007). Functional neural networks underlying response inhibition in adolescents and adults. Behav Brain Res 181: 12-22.

Stevens MC, Pearlson GD, Calhoun VD (2009). Changes in the interaction of resting-state neural networks from adolescence to adulthood. Hum Brain Mapp 30: 2356-2366.

Torrubia R, Avila C, Moltó J, Caseras X (2001). The Sensitivity to Punishment and Sensitivity to Reward Questionnaire (SPSRQ) as a measure of Gray's anxiety and impulsivity dimensions. Pers Individ Dif 31: 837-862.

Wetherill RR, Bava S, Thompson WK, Boucquey V, Pulido C, Yang TT et al (2012). Frontoparietal connectivity in substancenaive youth with and without a family history of alcoholism. Brain Res 1432: 66-73.

Wetherill RR, Castro N, Squeglia LM, Tapert SF (2013). Atypical neural activity during inhibitory processing in substance-naive youth who later experience alcohol-induced blackouts. Drug Alcohol Depend 128: 243-249.

Yarosh HL, Hyatt CJ, Meda SA, Jiantonio-Kelly R, Potenza MN, Assaf $M$ et al (2014). Relationships between reward sensitivity, risk-taking and family history of alcoholism during an interactive competitive fMRI task. PloS One 9: e88188.

Zachariou V, Klatzky R, Behrmann M (2014). Ventral and dorsal visual stream contributions to the perception of object shape and object location. J Cogn Neurosci 26: 189-209.

Zuckerman M, Neeb M (1979). Sensation seeking and psychopathology. Psychiatry Res 1: 255-264.

Supplementary Information accompanies the paper on the Neuropsychopharmacology website (http://www.nature.com/npp) 